\title{
Potenciando la equidad con docentes \\ latinoamericanos en entornos rurales bilingües: los proyectos de la Cátedra UNESCO - UV
}

\author{
Senent Sánchez, Joan María \\ Facultad de Filosofía y Ciencias de la Educación. Universidad de Valencia \\ senent@uv.es
}

\section{Resumen}

Durante los dos últimos años hemos desarrollado dos proyectos de la Cátedra Unesco-UV con la colaboración de universidades de Bolivia, Argentina, Perú y Paraguay y el equipo de la Universidad de Valencia. Ambos proyectos tienen en común el objetivo de elaborar materiales y recursos que los docentes puedan utilizar con facilidad en situaciones docentes complejas.

En el primero, el trabajo se ha dirigido a docentes de secundaria que actúan en la zona rural de la provincia de Salta (norte de Argentina), en situación de gran dificultad pues trabajan un área de estudio durante una semana con todos los alumnos de una escuela en una aldea, para dejar paso a otros compañeros que trabajan otras áreas las dos siguientes semanas y volver a la misma escuela tres semanas después. En el segundo proyecto, los recursos se han dirigido a docentes que trabajan en un contexto intercultural y bilingüe (español y guaraní, quechua, wichí o aymara) marcado por una fuerte situación de diglosia respecto a la lengua autóctona.

En ambos casos se busca modificar una fuerte situación de inequidad respecto a la acción docente, especialmente por carencia de formación y recursos de los propios docentes, y respecto al aprendizaje de los alumnos, marcado por la complejidad de un contexto bilingüe-diglósico y una carencia importante de instrumentos de trabajo.

La comunicación desarrolla el trabajo realizado, indicando la metodología seguida por los equipos de las diversas universidades y presenta los materiales elaborados y las previsiones de uso de dichos recursos.

\section{Abstract}

During last two years we have developed two projects of the Chair UNESCO - UV with the collaboration of universities of Bolivia, Argentina, Peru and Paraguay and the equipment(team) of the University of Valencia. Both projects have jointly the aim(lens) to elaborate materials and resources that the teachers could use easily in the very complex learning situations.

In the first one, the work has been directed to secondary teachers teachers that act in the rural zone of the province of Salta (north of Argentina), in situation of great difficulty since they are employed an area of study for one week with all the pupils of a school at a little village, to open way for other teaching working other areas two following weeks and to return to the same school three weeks later. In the second project, the resources have gone to teachers working in an intercultural and bilingual context (Spanish and Guarani, Quechuan, Wichí or Aymara) marked by a strong situation of diglosia with regard to the autochthonous language.

In both cases one seeks to modify a strong situation of inequity with regard to the educational action and the learning of the pupils. This paper explane the and presents the elaborated materials and the forecasts of use of the above mentioned resources.

Palabras clave: equidad, cooperación, recursos docentes, ruralidad, intercultural, bilingüismo.

Keywords: equity, rural context, intercultural, cooperation, educational resources, bilingualism.

\section{INTRODUCCIÓN}

Durante los últimos diez años hemos desarrollado en la Facultad de Filosofía y Ciencias de la Educación de la Universidad de Valencia, un programa específico de movilidad en América Latina para estudiantes de titulaciones de Educación (Pedagogía, Educación Social y en algunos casos Magisterio). El programa permite a los estudiantes realizar el último de los períodos de prácticas, 
durante seis meses, en América Latina y descansa en una estructura que solemos definir como un taburete de tres patas:

- Los estudiantes de educación de nuestra facultad y sus profesores.

- Una universidad latinoamericana con titulaciones de carácter socioeducativo (pedagogía, humanidades, trabajo social, educación social, ...) que incluyan períodos de prácticas.

- Una institución (asociación, ONG, fundación, proyecto, ...) que mantenga algún tipo de acuerdo con esa universidad y donde nuestros estudiantes puedan realizar las prácticas tutorizados por uno o varios profesores de la universidad americana.

Durante estos diez años hemos ido desarrollando esa estructura, eligiendo habitualmente universidades de tamaño mediano o pequeño y normalmente fuera de las capitales de los estados para favorecer la relación y la integración de los estudiantes. Así hemos ido desarrollando una red de una quincena de universidades desde México al sur de Chile a las que enviamos cada año 20-25 estudiantes habitualmente en parejas.

El seguimiento de las prácticas de estos estudiantes nos ha hecho ir estableciendo lazos profesionales y de amistad con colegas latinoamericanos, al tiempo que nos ha hecho conocer mucho mejor esas realidades socioeducativas donde realizan las prácticas nuestros estudiantes. Todo ello nos ha permitido diseñar y desarrollar proyectos tanto europeos como valencianos en el área de la cooperación, de la formación de docentes y educadores, de la animación sociocultural, etc, en la medida en que hemos ido conociendo más a fondo las características de esos entornos donde intervenían.

Algunas de esas experiencias han sido publicadas en diversos textos en los últimos años, Martínez (2011), Senent (2012), lo que ha ido configurando un proceso de retroalimentación teoría - práctica, que comenzaba habitualmente por esta última, se analizaba en un proceso teórico para dar lugar después a nuevas prácticas.

Fruto de esas prácticas, de su análisis teórico y de los lazos que íbamos creando con los profesionales de las universidades americanas, así como con los estudiantes que retornaron y alguno de los cuales lo hemos encontrado años después como compañero en la universidad, fuimos detectando, y compartiendo, problemáticas que tenía el profesorado de enseñanza obligatoria, muchos de los cuales se formaban en las universidades social del programa.

\section{PLANTEAMIENTO DEL TRABAJO}

La primera institución con la que iniciaos nuestra colaboración en américa Latina ya hace más de quince años fue la Universidad Nacional de Salta. Situada al norte de Argentina, con fronteras a Bolivia, Chile y Paraguay, la provincia es muy extensa, un tercio del territorio español, y la universidad, además de la sede central en Salta Capital, tiene dos o tres sedes más situadas a 400-500 km de la primera. Tanto esta provincia como la de Jujuy, destacan por la riqueza de etnias y de lenguas. Muchas comunidades de pueblos originarios conviven con criollos y mestizos, y en muchas de esas comunidades, lenguas como el guaraní, quechua, el chané y el wichí conviven con el castellano oficial.

Uno de los aspectos comunes que fuimos detectando en el análisis de la actuación de los docentes con quienes realizaban las prácticas nuestros alumnos era sin duda la ruralidad. En una provincia extensa, la mayor parte de las escuelas están en comunidades rurales, lo que lleva a desarrollar, en la educación primaria, muchas escuelas unitarias donde varios grados se concentran en la misma aula. En la educación secundaria, obligatoria también, las dificultades son mayores pues de una parte el alumnado es menor, por lo que está más desperdigado, y por otra la intervención docente no es generalista sino especializada, lo que fuerza la intervención de varios docentes sobre el mismo colectivo de alumnos. Esa situación fue el punto de partida de la Secundaria rural itinerante salteña. Organizada para que todos los alumnos tuvieran la opción de cursar la secundaria procurando restringir al máximo la necesidad de desplazarse, en un territorio donde los desplazamientos son difíciles y muchos estudiantes los han de hacer a pie o en caballería, establece cuatro profesores por escuela, cada una de los cuales imparte un área de trabajo: matemáticas-ciencias, lenguas, ciencias sociales y expresión plástica-artística-musical. Cada docente trabaja una semana al mes su área científica y se despide e los alumnos para volver un mes después. 
En ese contexto, donde aislamiento y falta de recursos se unen, resulta bastante complicado trabajar la capacitación de los docentes y por ello nuestro objetivo fue crear materiales que de forma sencilla pudieran trasladarse,- pensemos que muchos de los docentes realizan a pie buena parte de sus trayectos-, y aplicarse en una clase de secundaria poco numerosa (entre 5-12 alumnos).

Nos planteábamos como objetivo «unir la reflexión didáctica que hacemos en las aulas universitarias con la reflexión sobre la práctica docente recibida de diversas formas, especialmente en el medio rural y con especial incidencia en la secundaria rural itinerante, con las peculiaridades que tiene la enseñanza en ese entorno y que la diferencias de otros planteamientos didácticos secundarios. Sin duda las propias reflexiones y análisis de los docentes que usen este manual pedagógico precisarán su eficacia y nos ayudarán a desarrollarlo en el futuro» (Senent - De Anquin, 2017, 13)

Con este planteamiento diseñamos una Guía de Recursos que abordaba las siguientes temáticas con indicación de los autores que las trabajaron:

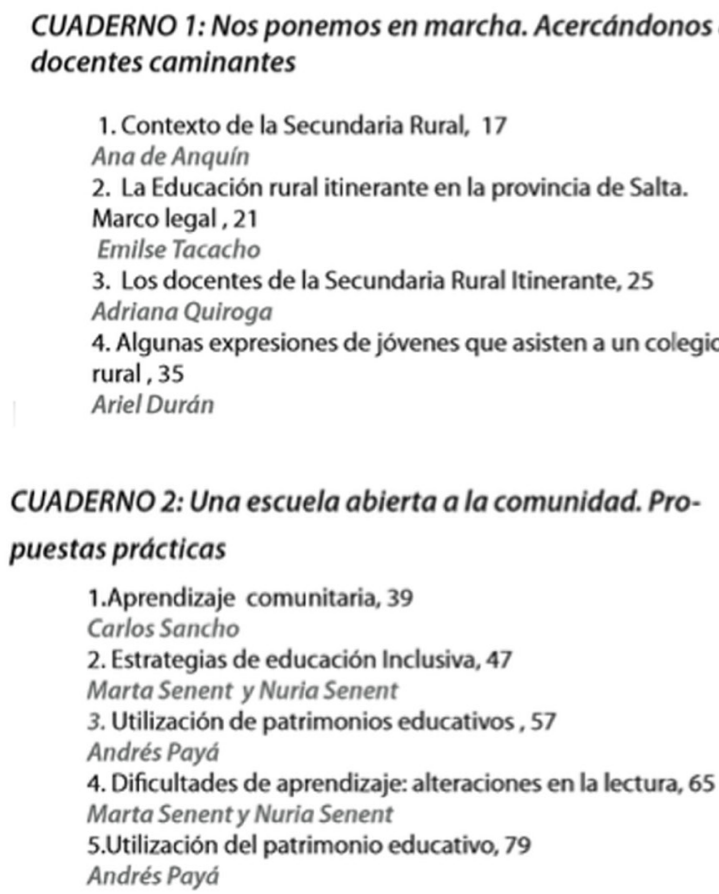

CUADERNO 3: Estrategias y proyectos que fomentan la cultura escolar

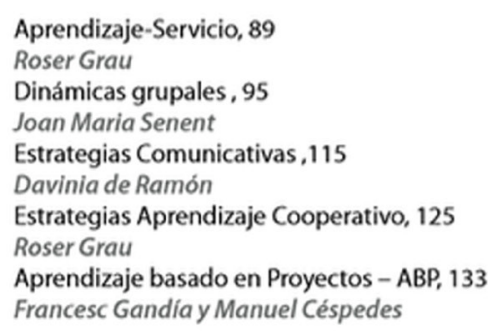

CUADERNO: Cerrando el proceso

Evaluación: Planteamientos y estrategias, 143

Ma Jesús Perales y Carlos Sancho

Pensar y vivir la escuela rural , 151

No es más que un hasta luego, 157

Joan María Senent

Anexos, 159

Instituciones y direcciones útiles para docentes rurales.

Nuestro siguiente paso fue establecer la forma de distribución de los recursos, en contacto con nuestros colegas de la Universidad de Salta y con el propio gobierno salteño. Se ha realizado de forma 
diferentes aprovechando viajes de miembros del grupo y se ha distribuido en institutos terciarios de formación de docentes, en reuniones de profesorado convocadas por la coordinación administrativa, en la universidad, en algunos grandes centros escolares con bastantes docentes y quizás lo que para mí ha sido más interesante han sido las tertulias o grupos de discusión sobre los recursos elaborados, pues en ellos hemos ido explicando la guía de recursos, justificando los diversos capítulos e instrumentos que ofrece, y al mismo tiempo para nosotros ha sido una revisión del proceso que sin duda ha estado presente en el siguiente proyecto.

El trabajo sobre el proyecto de creación de materiales para los docentes de la secundaria rural itinerante y su posterior distribución nos puso sobre la mesa una cuestión ya conocida por los años del programa específico de cooperación pero que trabajando con los docentes se hacía más evidente: muchos de ellos/as trabajan en contextos bilingües y claramente multiculturales lo que introduce un factor nuevo, que añade sin duda complejidad a su tarea, ya de por si bastante complicada.

En el caso argentino de la provincia de Salta, territorio a cuyos docentes destinamos la guía de recursos, la situación bilingüe se produce en la zona de Tartagal, cercana a la frontera con Bolivia, donde hay una sede de la Universidad Nacional de Salta. En esa zona ya habíamos estado en contacto con comunidades guaraníes, chanés y wichís a cuyas escuelas ya habían llegado nuestros alumnos/as del programa específico de movilidad y con algunos de los docentes ya habíamos compartido materiales y recursos no muy diferentes de los que utilizamos en un sistema bilingüe como el valenciano.

La constatación de la peculiaridad del trabajo de esos docentes nos llevó a consultar con otros colegas de instituciones con las que también tenemos convenio y que en algunos casos también reciben a nuestros alumnos. Ese fue el caso de la Universidad Nacional de Villarrica (Paraguay), del Instituto Superior Pedagógico CREA de Lima y de la Universidad Autónoma Nacional "Gabriel Moreno» de Santa Cruz (Bolivia), junto con la Universidad Nacional de Salta, con quien habíamos trabajado en el proyecto anterior. Asimismo hemos tenido colaboración de compañeras ecuatorianas de la región de Esmeraldas, más marcada en este cado por la multiculturalidad que por el bilingüismo.

Conjuntamente lanzamos un proyecto a la Cátedra Unesco-UV, para generar un nueva Guía de Recursos pedagógicos, pero esta vez centrados en docentes de primaria y secundaria que trabajaran en centros situados en contextos interculturales bilingües latinoamericanos, concretamente en contextos de lengua guaraní, quechua, wichí y aymara, en alguns casos como lenguas co-oficiales con el castellano y por tanto presentes en el curriculum tanto como materias a impartir como en algunos casos como lengua vehicular, en otros como lenguas presentes en las aulas aunque no tuvieran el rango de oficialidad, pero con el deseo de los docentes y las escuelas de usarlas en las clases y generar recursos para trabajar con ellas. Compartiendo todos los contextos una clara situación diglósica donde el castellano como lengua A tiene un status muy superior a las otras lenguas B, quizás no en su uso, pero si en cuanto a la presencia de materiales y recursos.

Aprobado el proyecto por la Universidad de Valencia, hemos tenido que trabajar de forma acelerado pues los proyectos de Cátedra Unesco se han de ejecutar en un año, de manera que constituimos en cada una de las sedes un equipo de trabajo, siendo el más numeroso el equipo de Valencia que ya estaba formado en el proyecto anterior y aun cuando inicialmente se pensó en que los equipos fueran internacionales trabajando vía Skype y mail, comprobamos después que en el plazo del proyecto, un año, que en realidad se convirtió en 10 meses por cuestiones administrativas, esto no era posible, por lo que manteniendo la comunicación entre los equipos optamos porque estos trabajarán de forma autónoma a partir de una primera distribución de los contenidos de la Guía.

Somos conscientes al acabar la Guía de recursos que las condiciones de tiempo y financiación solo nos permitían abordar algunas de las cuestiones que se plantean a un profesor en esos contextos bilingües e interculturales y que quedan muchas más que no hemos podido desarrollar, lo que nos servirá de motivación para nuevos proyectos, de igual modo que la difusión y el uso de los materiales creados nos planteará sin duda la revisión de los mismos y pondrá en cuestión más de un planteamiento que en la Guía hemos trabajado, obligando claramente a su revisión. 
Con todas esas cuestiones presentes y aceptando que nuestro trabajo entra en ese concepto de provisionalidad, que todo docente, especialmente en aulas y escuelas complejas, tiene en cuenta, la guía elaborada ha seguido el siguiente esquema:

\section{ÍNDICE}

1 Enseñar en entornos interculturales bilingūes: un reto apasionante - Joan 7 Maria Senent

\section{REFLEXIONES Y EXPERIENCIAS}

2 Identidad comunitaria y educación intercultural en contextos indigenas.

Roser Grau - Francesc Gandía

3 Contextos de la Educación Intercultural Bilingūe (EIB) en el noroeste de Argentina - Ana de Anguín

4 Crecer en equidad. Caminando hacia una educación en y para la igualdad de género en Esmeraldas (Ecuador) - Sonia Ortega, Zoraida Moncaya, Miriam Tudela

5 Bilingüismo y diglosia. Su impacto en el desarrollo infantil - Nuria Senent

6 La mediación cultural como recurso pedagógico para facilitar la educación intercultural: La experiencia del Proyecto Nau Social de la Unixersitat de València. Elisabeth Catalá - María Jesús Perales

7 El valor social de la educación como elemento de transmisión cultural Carlos Sancho, Ma Jesús Perales, Jesús Jornet

8 Construyendo una cultura de paz desde la escuela - Elisabeth Acha. Elizabeth Evans

9 Literatura Oral en espacios interculturales. Experiencias pedagógicas en la educación de Jóvenes y Adultos del Norte de Argentino - José Juarez

10 Qpaete reye yaeka ñande raikuere (todos juntos buscamos nuestra historia) - Adriana Quiroga, María Beatriz Bonillo, María Luisa Jalil, Marcelo Soria

11 Proyecto ciudadano - Pablo Zavala

12 Estrategias de comprensión lectora: propuesta para su implementación en el Nivel Primario de la Unidad Educativa de Basilio-Municipio La Guardia, de la ciudad de Santa Cruz, Bolivia - Victor Henry Andrade, María del Pilar Trigueros, Edith Victoria Villarroel, Rafael Melgar

\section{RECURSOS Y ESTRATEGIAS}

13 Revalorización y uso educativo de los juegos populares y tradicionales Andrés Payà

14 TIC y educación : Recursos y metodologías para la innovación educativa Davinia de Ramón

15 Educación para la salud - Aprender para vivir - Marta Senent

16 La mediación escolar como proceso de resolución de conflictos María Isabel Viana

17 Fortaleciendo la cultura guaraní: un reencuentro con los juegos tradicionales del Paraguay, Daxi Duarte, Sindulfo García, Norma Bamirez

18 Educar y trabajar para la sostenibilidad desde la educación ambiental Joan Maria Senent-Manuel Madolell

19 Fin de la vereda, inicio del camine- Joan Maria Senent 
Acabado el trabajo, viene ahora la cuestión de cómo explotar y utilizar los materiales creados. Hemos empezado los contactos con las universidades y docentes de los diversos territorios para implementar cursos de capacitación que desarrollen la formación de docentes desde ese ángulo de la interculturalidad y el bilingüismo. Hablamos de formación permanente para docentes que ya están en ejercicio y en colaboración con las universidades en las que trabajan miembros del equipo, así como con las autoridades que tienen bajo su responsabilidad la formación docente en esos territorios.

La vereda que iniciamos ha dado lugar a un camino, del que hemos andado los primeros tramos per que sin duda se abre y se adecua a cada situación a través de procesos de formación-capacitación. Esa es nuestra siguiente etapa, hemos creado durante dos años unos recursos que junto a otros creados por otras personas e instituciones nos gustaría aplicar a través de procesos de capacitación en los que intervengamos profesorado del equipo junto a otros profesionales y que sea organizado de forma diversa, de manera que se adecúe a las estructuras universitarias y de formación del profesorado de la región de Salta en Argentina, de Santa Cruz de la Sierra en Bolivia y de Lima y su entorno andino.

Lógicamente esto no es algo que el equipo de docentes que ha redactado este libro pueda hacer solo. Por ello hemos iniciado ya los primeros contactos con universidades y otras entidades para iniciar ese camino en el que nos gustaría contar con la financiación que algunas convocatorias públicas de cooperación al desarrollo puedan aportarnos. No habrá una única forma, sino que en función de los territorios será diversa y en colaboración con instituciones locales. Hemos iniciado los contactos para comenzar esos procesos en Salta, Santa Cruz y Lima, y posteriormente se podrían extender a otras zonas

Ese es el camino trazado para los próximos años y estos materiales creados en este cajón de recursos, junto con otros creados anteriormente, serán sin duda un empujón y un estímulo en ese camino de la formación docente en colaboración internacional que ahora comenzamos.

\section{REFERENCIAS BIBLIOGRÁFICAS}

Ansion, J. (2009). Educación superior e interculturalidad en el Perú. Apuntes preliminares. ISEES. Inclusión Social y Equidad en la Educación Superior, 4, 17-30.

Cieza, J.A. (2006). Educación Comunitaria. Revista de Educación, 339, 765-799.

De Anquín, A. (2008) Zona inhóspita. De maestros y pobladores, de lugares y tiempo. EUNSa.

Degregori, C.I. (1999). Estado nacional e identidades étnicas en Perú y Bolivia, en K.Koonings y P. Silva (editores), Construcciones étnicas y dinámicas sociocultural en América Latina, Quito: Abya Yala.

Grau Vidal, R C. Sancho Álvarez, M. García Carmona (2015). Iniciativas educativas innovadoras en escuelas interculturales bilingües. Experiencia en los pueblos originarios de Tartagal (Norte argentino). Ponencia.

Laura, María (2004). Estrategias didácticas en escuelas rurales de la provincia de Salta. Tesis de Maestria. UBA.

Leañez, M, Vara, O; Vera M. Durán. Procesos de reconocimientos: mujeres andinas como agentes de trasmisión de identidades/alteridades en las prácticas educativas y comunitarias. Ponencia.

López, L.E. y Küper, W. (1999). La educación intercultural bilingüe en América Latina: balance y perspectivas. Revista Iberoamericana de Educación, 20, 1-48.

Martínez Usarralde, MJ (2011): Sentipensar el sur. PUV. Valencia.

Planella, J. (2008). Educación social, acompañamiento y vulnerabilidad: hacia una antropología de la convivencia. Revista Iberoamericana De Educación, 46 (5), 1-14.

Senent, JM - De Anquín , A (Coord.) (2017): Abriendo Veredas. Guía de Recursos pedagógicos para docentes de la Secundaria Rural itinerante. Servei de Publicacions de la Universitat de Valencia. Valencia.

Senent, JM (2012): Escritos de la cooperación y la movilidad. Servei de Publicacions de la Universitat de Valencia. Valencia.

Trapnell, L. (2011). Desde la Amazonía peruana: aportes para la formación docente en la especialidad de educación inicial intercultural bilinguie. Educación, 20 (39), 37-50. 